\title{
Computer Aided Geometric Modeling of Solutions to the Tasks of Applied Cyclography
}

\author{
K.L. Panchuk, E.V. Lyubchinov, T.M. Myasoedova \\ panchuk_kl@mail.ru|lubchinov.e.v@yandex.ru|mtm44mtm44@mail.ru \\ Omsk State Technical University, Omsk, Russia
}

\begin{abstract}
In the present paper the solutions based on cyclographic method are considered on the example of two applied tasks: generation of road surface forms and pocket machining process engineering. Geometric structures based on cyclographic mapping of space E3 on plane $E_{2}$ and the corresponding mathematical models in the form of systems of parametric equations are provided. On the basis of the developed models, analytical solutions to the problems of shaping the surface and linear forms of the studied objects in the areas of road design and surface treatment of mechanical engineering products were obtained. The models develop the authors' previous research and are aimed at comprehensive solution to the problems of surface form generation in application to the two mentioned tasks.
\end{abstract}

Keywords: cyclographic method, mapping, mathematical modeling, roads, offset curves, pocket surfaces.

\section{Introduction}

Cyclography as a method of representation of space $E_{n}$ on space $E_{n-I}$ has appeared at the turn of XIX century owing to the research of West European geometers [6]. As shown by the vast body of research later conducted by Russian and foreign scientists, the cyclographic method, previously considered an instrument of theoretical analysis [3-5,10,13], proved effective in solution of various applied tasks of geometric optics $[10,12,16]$, Computer Aided Geometric Design (CAGD) $[11,12]$, road surface form modeling $[9,11]$, pocket machining of engineering products $[7,8]$, etc.

In the present paper, in further development of practical application of cyclographic method, the research results summarizing the authors' previous research in computer aided geometric modeling of road surface forms and process engineering of pocket machining are considered.

\section{Elements of theory of cyclographic modeling and solution of practical tasks on its basis}

\subsection{Computer aided geometric modeling of automotive road surface forms}

Formation of mathematical models of automotive road surfaces is an essential task of their design. Geometry of these surfaces has direct influence on traffic safety. Therefore, if the geometry does not conform to the current norms and regulations [1], it is not possible to cancel out this issue by means of various roadway coverings.

Modern mathematical modeling of road surface forms is based on several approaches. The most widely known of them are considered in papers $[2,17,18]$. These approaches are aimed at conforming to the current state regulations [1]. According to these regulations, road surface on straight road segments must have dual-slope profile consisting of segments of straight lines of certain constant incline, while on circular road segments road surface must have single-slope profile.

In the present paper geometric modeling of road surface forms is considered on the basis of cyclographic mapping of road axis. Road axis is considered given in the form of a smooth spatial curve:

$$
\bar{P}(t)=(x(t), y(t), z(t)), T_{0} \leq t \leq T,
$$

where functions $x(t), y(t), z(t)$ have continuous derivatives of up to the second order inclusive with respect to parameter $t$ within range $\left[T_{0}, T\right]$, at that, in every point within said range rank $\left\|x^{\prime}(t), y^{\prime}(t), z^{\prime}(t)\right\|=1$.

A cyclographic image of road axis as a spatial line constitutes an envelope of one-parameter multitude of bases of cones of revolution, vertices of which belong to road axis $\bar{P}(t)$. The equations of the envelope with half-angle of generatrix incline at the vertex $\beta=\beta(t)$ are of the following form [11]:

$$
\begin{aligned}
& x_{\beta}^{(1,2)}(t)=x(t)+\varepsilon(t) \cdot\left(\eta_{x}(t) \mp \delta_{x}(t)\right), \\
& y_{\beta}^{(1,2)}(t)=y(t)+\varepsilon(t) \cdot\left(\eta_{y}(t) \pm \delta_{y}(t)\right),
\end{aligned}
$$

where $\varepsilon(t)=z(t) \cdot e(t) ; \eta_{x}(t)=\frac{-x^{\prime}(t) \cdot \mu(t)}{\lambda(t)}$;

$$
\begin{aligned}
& \delta_{x}(t)=\frac{y^{\prime}(t) \sqrt{\lambda(t)-\mu^{2}(t)}}{\lambda(t)} ; \eta_{y}(t)=\frac{-y^{\prime}(t) \cdot \mu(t)}{\lambda(t)} ; \\
& \delta_{y}(t)=\frac{x^{\prime}(t) \sqrt{\lambda(t)-\mu^{2}(t)}}{\lambda(t)} ; \mu(t)=e(t) \cdot z^{\prime}(t)+e^{\prime}(t) \cdot z(t) ; \\
& \lambda(t)=x^{\prime}(t)^{2}+y^{\prime}(t)^{2} ; e(t)=\operatorname{tg}(\beta(t)) .
\end{aligned}
$$

Simultaneous solution of the equations (1) and (2) allows us to acquire a ruled surface, parametric equations of which have the following form:

$$
\begin{aligned}
& X(t, l)=x(t)+l \cdot\left[x_{\beta}^{(1,2)}(t)-x(t)\right], \\
& Y(t, l)=y(t)+l \cdot\left[y_{\beta}^{(1,2)}(t)-y(t)\right], \\
& Z(t, l)=z(t) \cdot(1-l) ; \\
& T_{0} \leq t \leq T, L_{0} \leq l \leq L .
\end{aligned}
$$

Curves with vertices belonging to the given curve $\bar{P}(t)$ are generated upon intersection of surface (3) by planes normal with respect to orthogonal projection of road axis $\bar{P}_{1}(t)=(x(t), y(t))$. The acquired profiles are different from the conventional ones conforming to current norms and regulations [1]. It is therefore necessary to transform the acquired ruled surface into a linear surface conforming to the norms. This transformation is considered in paper [11].

In order to provide required carriage way (roadside) width, initially, curves equidistant with respect to road axis orthogonal projection $\bar{P}_{1}(t)$ are constructed. Cylindrical surfaces projecting with respect to plane $(x y)$ are then constructed on these curves. Intersection of these surfaces and the transformed ruled surfaces results in generation of carriage way edges. The equations of these edges and road axis (1) allow us to acquire the sought ruled surfaces of carriage way. It is possible to acquire roadside surfaces bounded by carriage way edges and roadside edges in a similar fashion.

For the cases of segments straight and circular (superelevated) in map projection, half-angle $\beta$ in equations (2) is constant. As an example, a computer rendering of straight road segment acquired on the basis of cyclographic mapping is depicted on fig. 1 . 


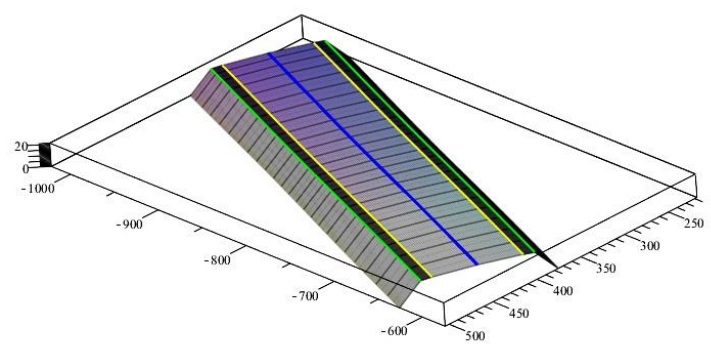

Fig.1. Computer aided cyclographic modeling of road surface forms on straight road segment

The transition segments are the most complex in road surface form design. The transition road segments have a particular feature of gradual change of cross profile from dual slope characteristic to straight segment to single slope characteristic to circular segment. Scheme of road surface generatrix incline variation on transition segment is presented on fig. 2.

For transition segments a function of half-angle variation $\beta$ $=\beta(t)$ is applied. The plot of half-angle variation function is depicted on fig. 3 ; it describes law of road surface generatrix incline variation with respect to parameter $t$ of road axis. The function must be continuous and single-valued within its domain of definition. The function must be smooth with no less than second order of smoothness in junction points in the interest of traffic safety [18].

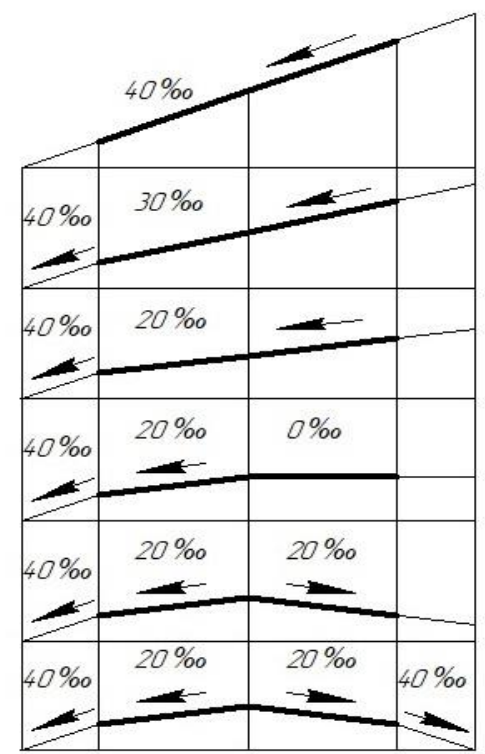

Fig.2. Scheme of road surface form generatrix incline variation on transition segment

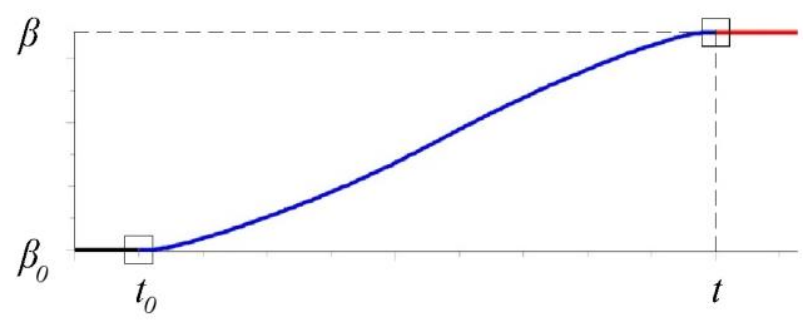

Fig.3. Plot of function of road surface form generatrix incline variation on transition segment
In paper [18] in order to achieve the required order of smoothness of junction of carriage way edges and roadside edges it is proposed to apply the following spline function of the fifth order as a function of incline variation:

$$
\begin{aligned}
& \beta(t)=\beta_{0}+a_{1}\left(t-t_{0}\right)+a_{2}\left(t-t_{0}\right)^{2}+a_{3}\left(t-t_{0}\right)^{3}+ \\
& +a_{4}\left(t-t_{0}\right)^{4}+a_{5}\left(t-t_{0}\right)^{5} .
\end{aligned}
$$

By combination of the expressions (4) and (2) and by performing certain necessary rearrangements, the equations of carriage way edges for transition segment and, correspondingly, the sought road surface form with variable generatrix incline are acquired. The result of computer aided modeling of transition segment surfaces on the basis of cyclographic mapping is presented on fig. 4.

In conclusion, the overall road surface form is divided into a number of structural segments: straight in map projection, circular (superelevated) and transition. A mathematical model taking into account essential features of road surface form generation is defined for each corresponding road segment. Calculation and rendering of road surface form on each road segement is readily realized by computer aided algebra systems due to parametric form of model representation.

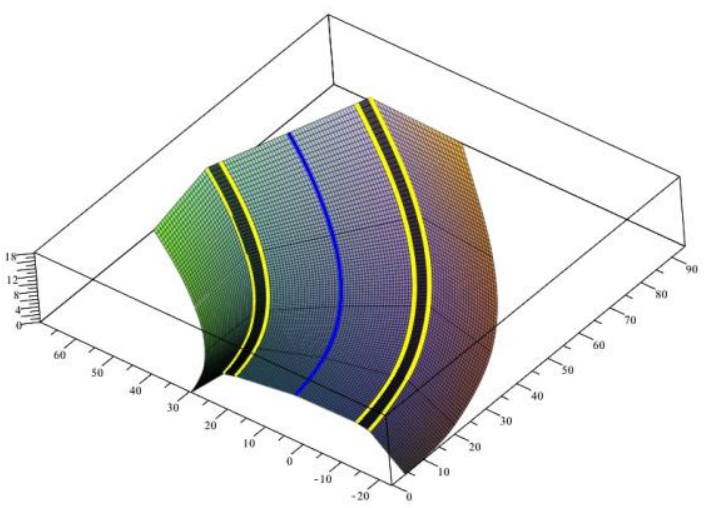

Fig. 4. Computer aided cyclographic modeling of road surface forms on transition road segment

Fig. 5 represents the compound result of computer aided road surface form modeling.

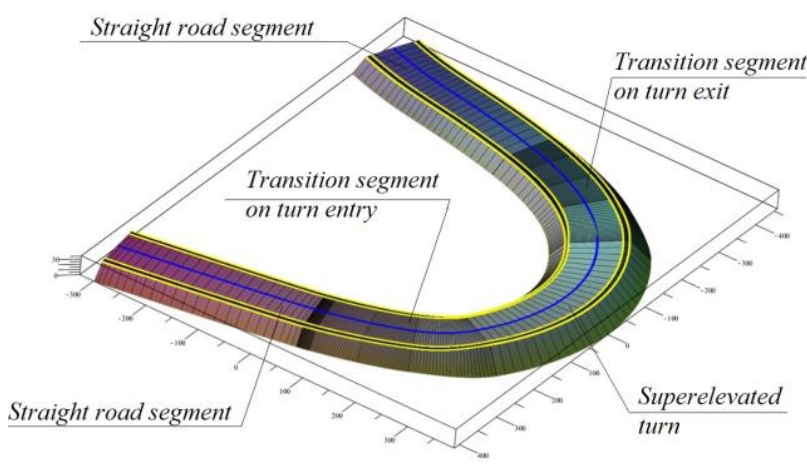

Fig.5. Compound cyclographic model of road surface form

\subsection{Computer aided geometric modeling in pocket machining process engineering}

Pocket machining process engineering on $\mathrm{NC}$ units requires cutting tool trajectory calculation and optimization. A cutting tool moves along equidistant trajectories called "offset curves" $(O C)$, gradually approaching the workpiece contour as it cuts. An equidistant curve is a curve of fixed distance from a given curve. The problem of optimization of $O C$ generation includes the following tasks: analysis and trimming of non-working $O C$ 
segments and analysis and trimming of opposing $O C$ of internal (island) and external boundary contours [4, 5, 7].

$O C$ family modeling for multiply connected area bounded by closed contours of pocket surfaces is performed on the basis of cyclographic mapping of Euclidean space. The modeling is considered in the view of Medial Axis Transform (MAT) [16].

$M A T$ is a spatial curve reconstructed in space on the basis of cyclographic mapping of geometric information on flat area and its boundary contour. MAT is generated upon intersection of modeled $\alpha$-surfaces, generatrices of which are inclined to the area on angle $\alpha=45^{\circ}$.

Pocket surface with islands in plane $(x y)$ is shaped by closed curvilinear contours consisting of arches of curves subsequently connected end-to-end with order of smoothness $C^{2}$ (fig.6). External boundary contour $a\left(a_{1}, \ldots, a_{n}\right)$ is described by equations of curves $a_{i}(i=1 . . n)$, of which it consists:

$$
a_{i}: \quad \bar{r}_{a i}=\left(x_{a}\left(t_{i}\right), y_{a}\left(t_{i}\right)\right), t_{i} \in R .
$$

Internal contour $b\left(b_{1}, \ldots, b_{k}\right)$ consists of arcs of curves $b_{j}(j=1 \ldots k)$ :

$$
b_{j}: \bar{r}_{b j}=\left(x_{b}\left(t_{j}\right), y_{b}\left(t_{j}\right)\right), t_{j} \in R .
$$

Internal contour $c\left(c_{1}, \ldots, c_{u}\right)$ consists of arcs of curves $c_{u}(u=1 \ldots m)[8]$ :

$$
c_{u}: \bar{r}_{c u}=\left(x_{c}\left(t_{u}\right), y_{c}\left(t_{u}\right)\right), t_{u} \in R .
$$

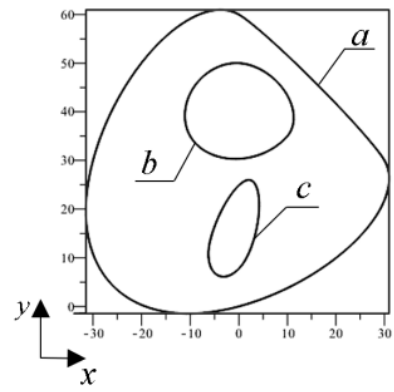

Fig. 6. The initial curvilinear contours of pocket $a$ and islands $b$ and $c$

With the help of expressions (8) it is possible to acquire parametric equations of evolutes of composite curves $a, b$, and $c$. For $e_{a}$ : $\left(x_{e a}\left(t_{i}\right), y_{e a}\left(t_{i}\right)\right), e_{b}:\left(x_{e b}\left(t_{j}\right), y_{e b}\left(t_{j}\right)\right)$, and $e_{c}$ : $\left(x_{e c}\left(t_{u}\right), y_{e c}\left(t_{u}\right)\right)$ correspondingly:

$$
\begin{gathered}
e_{a i}: \bar{r}_{e a}\left(t_{i}\right)=\bar{r}_{a i}+R_{a i} \bar{n}_{a i}, \\
e_{b j}: \bar{r}_{e b}\left(t_{j}\right)=\bar{r}_{b j}+R_{b j} \bar{n}_{b j}, \\
e_{c u}: \bar{r}_{e c}\left(t_{u}\right)=\bar{r}_{c u}+R_{c u} \bar{n}_{c u},
\end{gathered}
$$

where $R_{a i}, R_{b j}$ и $R_{c u}$ represent curvature radiuses; $\bar{n}_{a i}, \bar{n}_{b j}$, and $\bar{n}_{c u}$ represent unit vectors of curve normals. For evolutes $e_{a i}, e_{b j}$, and $e_{c u}$ let us construct their spatial images - curves $m_{a i}$, $m_{b j}$, and $m_{c u}$ - keeping in mind that applicate $z$ is negative for internal contours:

$$
\begin{aligned}
& m_{a i}: \bar{r}_{m a}\left(t_{i}\right)=\left(x_{e a}, y_{e a}, z_{e a}\right), \\
& m_{b j}: \bar{r}_{m b}\left(t_{j}\right)=\left(x_{e b}, y_{e b}, z_{e b}\right), \\
& m_{c u}: \bar{r}_{m c}\left(t_{u}\right)=\left(x_{e c}, y_{e c}, z_{e c}\right),
\end{aligned}
$$

where $z_{e a}=+\sqrt{\left(x_{a i}-x_{e a}\right)^{2}+\left(y_{a i}-y_{e a}\right)^{2}}$,

$$
z_{e b}=-\sqrt{\left(x_{b j}-x_{e b}\right)^{2}+\left(y_{b j}-y_{e b}\right)^{2}}, z_{e c}=-\sqrt{\left(x_{c u}-x_{e c}\right)^{2}+\left(y_{c u}-y_{e c}\right)^{2}}
$$

$\alpha$-surfaces $P_{a i}, P_{b j}$, and $P_{c u}$ are formed by pairs of curves $a_{i}$ and $m_{a i}, b_{j}$ and $m_{b j}, c_{u}$ and $m_{c u}$, serving as generatrices (fig. 7):

$$
\begin{array}{ll}
P_{a i}: & \bar{r}_{P i}\left(t_{i}, l\right)=\bar{r}_{m a}\left(t_{i}\right)+l_{i}\left(\bar{r}_{a}\left(t_{i}\right)-\bar{r}_{m a}\left(t_{i}\right)\right), \\
P_{b j}: & \bar{r}_{P j}\left(t_{j}, l_{j}\right)=\bar{r}_{m b}\left(t_{j}\right)+l_{j}\left(\bar{r}_{b}\left(t_{j}\right)-\bar{r}_{m b}\left(t_{j}\right)\right),
\end{array}
$$

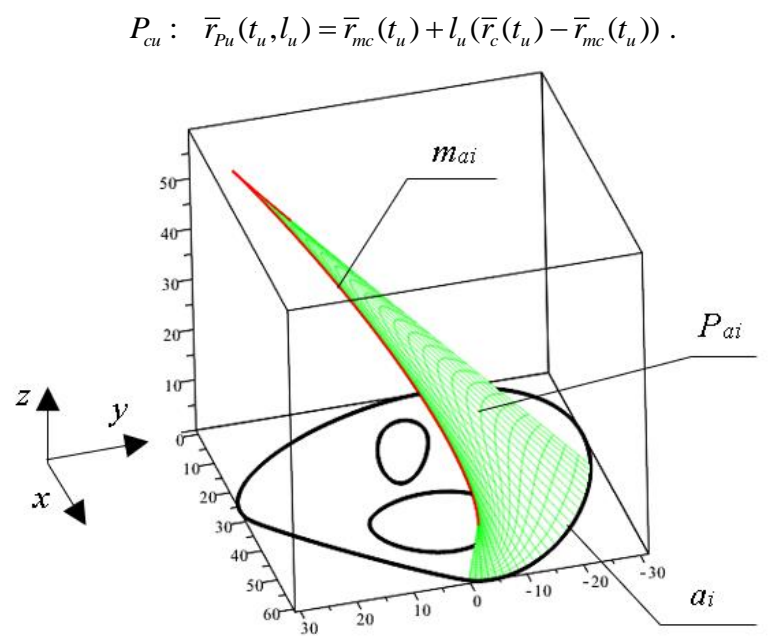

Fig.7. $\alpha$-surface formation

Geometric MAT is generated as a composite spatial curve of pairwise intersection of $\alpha$-surfaces (fig.8).

$$
M A T=\left(P_{a i} \cap P_{b j}\right) \bigcup\left(P_{a i} \cap P_{c u}\right) \bigcup\left(P_{b j} \cap P_{c u}\right) .
$$

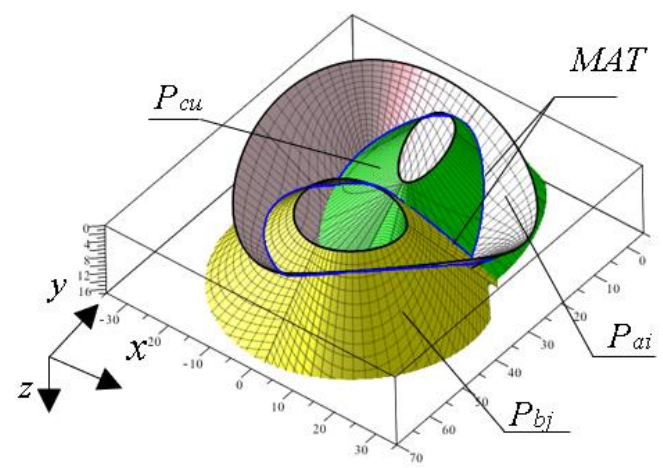

Fig.8 $\alpha$-surfaces of external and internal contours:

$$
P_{a i}, P_{b j}, P_{c u} \text { and } M A T
$$

By pairwise intersection $\alpha$-surfaces generate curves $s_{i}$. A combination of curves $s_{i}$ belonging to $\alpha$-surfaces constitutes a continuous multitude of points, which are $\alpha$-images of points of intersection between the $\alpha$-surfaces and plane (xy). If $s_{i} \in P_{a i}$, then curve $a_{i}$ is an $\alpha$-projection (image) of curve $s_{i}$. Correspondingly, if $s_{i} \in P_{b j}$, then curve $b_{j}$ is an $\alpha$-projection of curve $s_{i}$ and if $s_{i} \in P_{c u}$, then curve $c_{u}$ is an $\alpha$-projection (image) of curve $s_{i}$.

Therefore, curves $a_{i}, b_{j}$, and $c_{u}$ constitute branches of one common curve of intersection between plane $(x y)$ and an envelope of one-parameter multitude of $\alpha$-cones with vertices on $s_{i}$ and axes perpendicular to plane (xy). Base of each of the cones in plane (xy) is a circle of radius $R$ tangent to curves $a_{i}, b_{j}$, $c_{u}$, or $a_{i}, c_{u}$, or $a_{i}, b_{j}$, or $b_{j}, c_{u}$. Therefore, in plane $(x y)$ a continuous multitude of points $(x, y, R=z)$ constituting centers of circles of radiuses $R=z$ is generated. Obviously, this multitude of points and circles represents MAT.

The acquired $\alpha$-surfaces are sectioned by means of a horizontal bundle of planes along the $z$ axis with step $\Delta z_{i}=h_{i}=\delta=$ const. These lines are subject to analysis with subsequent trimming of non-working segments according to $M A T$. By means of orthogonal projection on plane (xy), the acquired level curves generate a family of $O C$ (fig.9). Level lines of the internal contours $L_{i n b(i, j)}, L_{i n c}(i, u)$ level lines of the external contour $L_{e x t}(i, k)$ are defined by the following parametric equations correspondingly:

$$
\bar{r}_{i n b(i, j)}=\left(x_{i n b(i, j)}\left(h_{i}, \tau_{j}\right), y_{i n b(i, j)}\left(h_{i}, \tau_{j}\right), z_{i n b(i, j)}\left(h_{i}, \tau_{j}\right)\right) \text {, }
$$




$$
\begin{aligned}
& \bar{r}_{\text {inc }(i, u)}=\left(x_{\text {inc }(i, u)}\left(h_{i}, \tau_{u}\right), y_{\text {inc }(i, u)}\left(h_{i}, \tau_{u}\right), z_{\text {inc }(i, u)}\left(h_{i}, \tau_{u}\right)\right),(11) \\
& \bar{r}_{\text {ext }(i, k)}=\left(x_{\text {ext }(i, k)}\left(h_{i}, \tau_{k}\right), y_{\text {ext }(i, k)}\left(h_{i}, \tau_{k}\right), z_{\text {ext }(i, k)}\left(h_{i}, \tau_{k}\right)\right),
\end{aligned}
$$

where $i$ represents the index of sectional plane; $\tau_{j}, \tau_{u}$, and $\tau_{k}$ represent parameters of shape of segments $L_{i n b(i, j)}, L_{i n c(i, j)}$, and $L_{\text {ext }(i, k) \text {. }}$

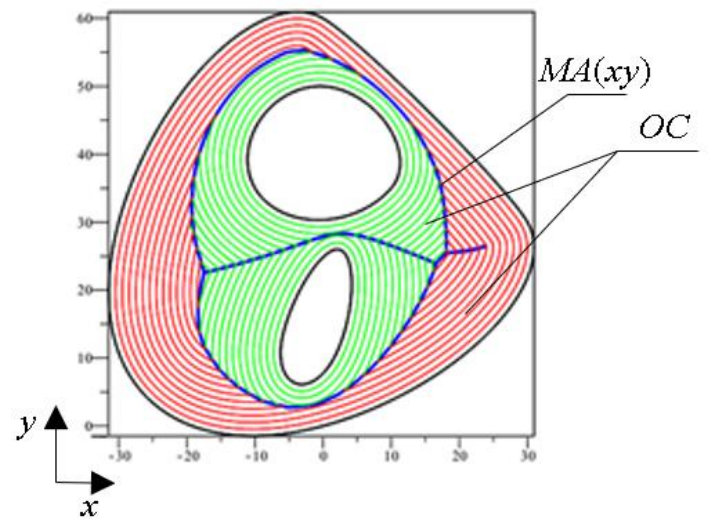

Fig.9. A family of offset curves $O C$ and medial axis $M A(x y)$

Orthogonal projection of MAT on plane ( $x y)$ is a curve, which constitutes medial axis $M A$.

The proposed geometric solution to the problem of $O C$ family generation for multiply connected area with curvilinear boundary contours in plane (xy) is different from the known algebraic solution $[4,5]$ in the following:

1. The proposed solution features a vivid graphic representation of all the multitude of geometric objects and model conditions in interrelation and interconnection with each other in virtual electronic space.

2. Mathematical model of the proposed geometric model of $O C$ family generation features parametric form of representation of its equations.

The mentioned differences make it significantly easier to acquire and analyze the solution to the task of $O C$ family generation, which facilitates control programming for pocket machining on $\mathrm{NC}$ units.

\section{Conclusion}

The considered cyclographic solutions to two urgent and diverse applied tasks confirm practicality and unveil potential of cyclographic modeling method.

\section{References}

[1] Automotive roads: Construction Norms and Rules 2.05.0285 Introduced 1987-01-01. Gosstroy of USSR. Moscow, TSITP Gosstroya USSR, 1986.

[2] Boykov V. N., Fedotov G. A., Purkin V. I. Automated Automotive Road Design (on the example of IndorCAD/Road) Moscow, MADI, 2005

[3] Choi H.I., Han C.Y., Moon H.P ., Roh K.H., Wee N.S.: Medial axis transform and offset curves by Minkowski Pythagorean hodograph curves, Computer-Aided Design 31 (1999), 5 p.p. 9-72.

[4] Choi H. I., Choi S. W., and Moon H. P. Mathematical theory of medial axis transform. Pacific J. Math., 181(1):56-88, 1997.

[5] Cho H.Ch., Choi H.I., Kwon S.-H., Lee D.S. and Wee N.S. Clifford algebra, Lorentzian geometry and rational parametrization of canal surfaces. Computer Aided Geometric Design, 21:327-339, 2004.

[6] Dr. Emil Muller. Vorlesungenüber Darstellende Geometrie. II. Band: Die Zyklographie. Edited from the manuscript by Dr. Josef Leopold Krames. Leipzig and Vienna, Franz Deuticke, 1929. - 476 pp.

[7] Held M 1991 On the computational geometry of pocket machining Lect. Notes in Comp. Sci. p 184.

[8] Myasoedova T. M., Panchuk K. L. Geometric model of generation of family of contour-parallel trajectories (equidistant family) of a machine tool. // IOP Conf. Series: Journal of Physics: Conf. Series. -2019. - vol. 1210(1). p. 012104. doi: 10.1088/1742-6596/1210/1/012104

[9] Panchuk K. L., Lyubchinov E.V., Myasoedova T.M. Cyclography. Aspects of Theory and Practical Applications. GraphiCon 2018: 28th The 28th International Conference on Computer Graphics and Vision. Conf. Proceedings. Tomsk, Tomsk Politechnical Univ., 2018. pp. 336 - 340.

[10] Panchuk K. L., Kaygorodtseva N. V. Cyclographic Desctiptive Geometry. Omsk, OmGTU, 2017232 p.

[11] Panchuk K.L., A. S. Niteyskiy, E. V. Lyubchinov. Cyclographic Modeling of Surface Forms of Highways. IOP Conf. Series: Materials Science and Engineering 262 (2017) 012108. doi:10.1088/1757-899X/262/1/012108

[12] Panchuk K. L., Lyubchinov E. V., Krysova I. V.Surface triads with optical properties. IOP Conf. Series: Journal of Physics: Conf. Series 944 (2018) 012086. doi :10.1088/1742-6596/944/1/012086

[13] Peternell M. Rational two-parameter families of spheres and rational offset surfaces. J. Symbolic Computation 45 (2010), 1-18.

[14] Peternell M., Pottmann H. Computing rational parametrizations of Canal Surfaces. J. Symbolic Computation 23 (1997), 255-266.

[15] Pottmann H., Peternell M. Applications of Laguerre Geometry in CAGD, Comp. Aided Geometric Design 15 (1998), 165-186.

[16] Pottmann H., Wallner J. Computational Line Geometry. Berlin. Heidelberg: Springer Verlag, 2001. 565 p.

[17] Purkin V. I. Basic Automated Design of Automotive Roads. Moscow, MADI, 2000.

[18] Salkov N.A. Modeling of Geometric Forms of Motor Roads. Moscow, INFRA-M, 2012. 